\title{
Distributed Control for Multiagent Consensus Motions with Nonuniform Time Delays
}

\author{
Mengji Shi and Kaiyu Qin \\ School of Aeronautics and Astronautics, University of Electronic Science and Technology of China, Chengdu 611731, China \\ Correspondence should be addressed to Kaiyu Qin; qinkaiyuuestc@sohu.com
}

Received 23 February 2016; Accepted 19 May 2016

Academic Editor: Zhan Shu

Copyright ( 2016 M. Shi and K. Qin. This is an open access article distributed under the Creative Commons Attribution License, which permits unrestricted use, distribution, and reproduction in any medium, provided the original work is properly cited.

\begin{abstract}
This paper solves control problems of agents achieving consensus motions in presence of nonuniform time delays by obtaining the maximal tolerable delay value. Two types of consensus motions are considered: the rectilinear motion and the rotational motion. Unlike former results, this paper has remarkably reduced conservativeness of the consensus conditions provided in such form: for each system, if all the nonuniform time delays are bounded by the maximal tolerable delay value which is referred to as "delay margin," the system will achieve consensus motion; otherwise, if all the delays exceed the delay margin, the system will be unstable. When discussing the system which is intended to achieve rotational consensus motion, an expanded system whose state variables are real numbers (those of the original system are complex numbers) is introduced, and corresponding consensus condition is given also in the form of delay margin. Numerical examples are provided to illustrate the results.
\end{abstract}

\section{Introduction}

As one of the fundamental problems in the study of the cooperative control for multiagent systems, consensus problem has received a fair amount of recent attention, because of its applications in formation control [1], target tracking [2], and sensor networks [3]. When researching consensus problems, time delay is hardly a matter of rarity, and numerous examples [4-16] have attested to its prevalence. It then raises a question: are the consensus conditions for the nondelayed systems still applicable in presence of time delays?

The treatments of systems with time delays have been widely studied, and researchers have developed diverse applicable approaches to analyzing the system stability under the occurrence of time delays, such as Lyapunov approach, the approach based on the properties of nonnegative matrices, and frequency domain approach. Lyapunov approach is a powerful tool of time domain analysis, with which one can easily handle nonlinearities and time-varying uncertainties. The consensus problems of delayed systems in $[4,5,7,8,10$, $12,13,16]$ have been worked out by establishing Lyapunov functions (functionals). Approaches based on the properties of nonnegative matrices can be available to the cases of dynamically changing topologies [15], but they are commonly limited to discrete-time systems. Linear delay systems are discussed in greater length with frequency domain approaches with which $[6,9,11]$ have studied the stability of multiagent systems with time delays, and necessary and sufficient conditions for these systems to reach consensus have been derived. Usually, consensus conditions derived by Lyapunovbased approaches are more likely to be conservative than the ones obtained by frequency domain approaches.

Taking nonuniform time delays into account, the paper investigates the consensus problems of multiagent systems in terms of motion. We consider two types of consensus motions: one is rectilinear motion, and the other is rotational motion. In the two systems, the agents aim to collectively move at a uniform velocity and angular speed, respectively, depending on their initial states. These multiagent models have found various applications, such as formation control for unmanned aerial vehicles (UAVs), spacecraft docking, and circular mobile sensor networks. The second order control algorithms are extended from the previous works [11, 17], but nonuniform time delays are considered.

An efficient frequency domain approach is employed to derived sufficient conditions for these consensus problems. The results are presented in the form of "delay margins." Each 
delay margin is a derived value that if all the time delays in the corresponding system are bounded by this value the system will reach consensus, but if all the delays exceed this value the agents will not achieve collective motion. Consequently, one cannot find out a greater value that if all the delays are bounded by that value the system still reaches consensus always. In [18], the author discussed the stabilities of secondorder multiagent systems with nonuniform delays using Lyapunov methods, and the results are presented in the form of Linear Matrix Inequalities (LMIs), which are different from our work. The previous publications [11,19] have studied multiagent systems with unstable modes, but nonuniform delays have not been considered. Reference [17] has proposed the rotating consensus algorithm, without consideration of delay effects. The rotating consensus algorithm we adopt enables the agents to achieve rotational consensus motion in the complex plane. However, in many cases, complex numbers cannot be processed by the processor. We expand the original system by separating the real part and imaginary part of each state variable. The new system has real state variables and coefficient matrices. We finally provide consensus conditions for the expanded system in the form of delay margin.

The remainder of this paper is organized as follows. Section 2 presents some mathematical preliminaries for the later proof, and with their help, we define the consensus problems and then protocols are given for the second-order multiagent systems with multiple time delays. Section 3 shows the stability analysis of the systems and discusses what will happen to the system to achieve rectilinear consensus motion if the input delays are absent; this section also provides an expansion to the system to reach rotating consensus, and this additional result allows us to simulate the algorithms in an easier way. Section 4 gives numerical simulations. Finally in Section 5, some conclusions are drawn.

\section{Model}

Before presenting the model, we first provide a brief narrative on some definition and results in graph theory, which are used extensively in the rest of this paper.

Consider a multiagent system consisting of $n$ agents. The communication network topology among them is represented by an undirected graph $\mathscr{G}(\mathscr{V}, \mathscr{E}, \mathscr{A})$, which consists of a set of nodes $\mathscr{V}=\left\{s_{i}\right\}, i \in \mathscr{I}=\{1,2, \ldots, n\}$, a set of edges $\mathscr{E} \subseteq \mathscr{V} \times \mathscr{V}$, and a weighted adjacency matrix $\mathscr{A}=\left[a_{i k}\right]$, where $a_{i i}=0$, and $a_{i k}=a_{k i} \geq 0$ (for $\mathscr{G}$ is undirected). $a_{i k}>0$ if and only if there exists an edge $e_{i k} \in \mathscr{E}$ between the $i$ th and $k$ th nodes, which implies that they can get information from each other. The set of neighbours of node $s_{i}$ is denoted by $N_{i} \triangleq\left\{s_{k} \in \mathscr{V}: e_{k i} \in \mathscr{E}\right\}$. The Laplacian associated with the graph $\mathscr{G}$ is defined as $L_{\sigma} \triangleq\left[\ell_{i k}\right]$, where $\ell_{i i}=\sum_{k=1}^{n} a_{i k}$ and $\ell_{i k}=-a_{i k}, i \neq k$. A path is a sequence of ordered edges $e_{r_{1} r_{2}}, e_{r_{2} r_{3}}, \ldots$, where $e_{r_{i} r_{i+1}} \in \mathscr{E}$. If there is a path between every pair of nodes in graph $\mathscr{G}$, the graph is said to be connected. The lemma below is given by [20].

Lemma 1. If the undirected graph $\mathscr{G}$ is connected, then its Laplacian $L_{\sigma}$ has one singleton zero eigenvalue (with eigenvector 1), and the rest of $n-1$ eigenvalues of $L_{\sigma}$ are all positive.
Consider $n$-agent systems with second-order dynamics:

$$
\begin{gathered}
\dot{x}_{i}(t)=v_{i}(t), \\
\dot{v}_{i}(t)=u_{i}(t),
\end{gathered}
$$

where $x_{i}, v_{i}$ denote the position and velocity of the $i$ th agent, respectively, and $u_{i}(t)$ is the control input. Denoting the state vector $\zeta_{i}(t) \triangleq\left[x_{i}(t), v_{i}(t)\right]^{T}$, we assume that the initial condition is $\zeta_{i}(p)=\zeta_{i}(0)$, for $p \in(-\infty, 0]$.

Two goals have been set for the system to achieve: rectilinear consensus motion and rotational consensus motion. Clearly, the control input $u_{i}(t)$ decides the behavior of the agents. We will propose two algorithms, respectively, for the two goals; before that, we use mathematical symbols to represent what will happen to the agents when the goals are achieved.

For the first goal, rectilinear consensus motion, if $\lim _{t \rightarrow+\infty}\left[\zeta_{i}(t)-\zeta_{k}(t)\right]=0$ is satisfied for all $i, k \in \mathscr{I}$, the control input $u_{i}(t)$ is said to solve the consensus problem asymptotically [11]. System (1) under this control input is referred to as "system $Y_{1}$ " hereinafter. In fact, in some cases (very extreme cases), the agents will not move collectively but all stay at a point as a final state; it depends on the initial conditions.

For the second goal, rotational consensus motion, the consensus is reached if

$$
\begin{aligned}
& \lim _{t \rightarrow+\infty}\left[v_{i}(t)-v_{k}(t)\right]=0, \\
& \lim _{t \rightarrow+\infty}\left\{\left[x_{i}(t)+w^{-1} j v_{i}(t)\right]-\left[x_{k}(t)+w^{-1} j v_{k}(t)\right]\right\} \\
& \quad=0, \\
& \lim _{t \rightarrow+\infty}\left[\dot{v}_{i}(t)-j w v_{i}(t)\right]=0
\end{aligned}
$$

for all $i, k \in \mathscr{I}$, where $w \in \mathbb{R}^{+}$is the constant angular velocity in which all agents move around a common point together [17]. We assume $w=1$ to simplify the future discussion. System (1) under a control input to satisfy (2) is called "system $Y_{2}$ " hereunder.

The state variables of agents in system $Y_{1}$ are considered in the real domain for convenience of calculation; that is, $x_{i}, v_{i} \in \mathbb{R}$ in $Y_{1}$. However, the agents in system $Y_{2}$ are intended to achieve rotational consensus motion in the complex plane; thus the state variables $x_{i}, v_{i} \in \mathbb{C}$ are complex values for system $Y_{2}$.

Taking nonuniform time delays into account, we modify the corresponding protocols proposed in $[11,17]$ and give the control inputs of our systems $Y_{1}$ and $Y_{2}$ as

$$
\begin{aligned}
& \text { For } Y_{1}: u_{i}(t)=\sum_{s_{k} \in N_{i}} a_{i k}\left\{k_{1}\left[x_{i}\left(t-\tau_{i k}\right)-x_{k}\left(t-\tau_{i k}\right)\right]\right. \\
& \left.\quad+k_{2}\left[v_{i}\left(t-\tau_{i k}\right)-v_{k}\left(t-\tau_{i k}\right)\right]\right\}, \\
& \text { For } Y_{2}: u_{i}(t)=j v_{i}(t)-\sum_{s_{k} \in N_{i}} a_{i k}\left[v_{i}\left(t-\tau_{i k}\right)\right. \\
& \left.\quad-v_{k}\left(t-\tau_{i k}\right)\right]
\end{aligned}
$$




$$
\begin{aligned}
- & \sum_{s_{k} \in N_{i}} a_{i k}\left\{\left[x_{i}\left(t-\tau_{i k}\right)+j v_{i}\left(t-\tau_{i k}\right)\right]\right. \\
& \left.-\left[x_{k}\left(t-\tau_{i k}\right)+j v_{k}\left(t-\tau_{i k}\right)\right]\right\},
\end{aligned}
$$

for any $i, k \in \mathscr{I}$, where $a_{i k}>0$ denotes the edge weight, and $\tau_{i k}=\tau_{k i}$ is the time delay for the $i$ th agent to get the state information of the $k$ th agent. We also consider the system to have $M$ different time delays, denoted by $\tau_{m} \in\left\{\tau_{i k}, i, k \in\right.$ $\mathscr{I}\}(m=1,2, \ldots, M)$.

We use matrix notation to write the models of both systems in compact form. To unify the form, we define

$$
\begin{aligned}
& \psi_{1}(t) \\
& \triangleq\left[x_{1}(t), v_{1}(t), x_{2}(t), v_{2}(t), \ldots, x_{n}(t), v_{n}(t)\right]^{T}, \\
& \psi_{2}(t) \triangleq\left[x_{1}(t), c_{1}(t), x_{2}(t), c_{2}(t), \ldots, x_{n}(t), c_{n}(t)\right],
\end{aligned}
$$

where $c_{i}(t)=x_{i}(t)+j v_{i}(t)$ for all $i \in \mathscr{I} \cdot \psi_{1}(t)$ and $\psi_{2}(t)$ are the state vectors of system $Y_{1}$ and $Y_{2}$, respectively. By defining coefficient matrices

$$
\begin{aligned}
& A_{1} \triangleq\left[\begin{array}{ll}
0 & 1 \\
0 & 0
\end{array}\right], \\
& B_{1} \triangleq\left[\begin{array}{ll}
0 & 0 \\
k_{1} & k_{2}
\end{array}\right], \\
& A_{2} \triangleq\left[\begin{array}{cc}
j & -j \\
0 & 0
\end{array}\right], \\
& B_{2} \triangleq\left[\begin{array}{cc}
0 & 0 \\
-1 & 1+j
\end{array}\right],
\end{aligned}
$$

system (1) under the control inputs (3) can be rewritten as

$$
\begin{aligned}
Y_{q}: \dot{\psi}_{q}(t)= & \left(I_{n} \otimes A_{q}\right) \psi_{q}(t) \\
& -\sum_{m=1}^{M}\left(L_{\sigma m} \otimes B_{q}\right) \psi_{q}\left(t-\tau_{m}\right),
\end{aligned}
$$

for $q=1,2$, with the initial condition $\psi_{q}(p)=\psi_{q}(0), p \in$ $(-\infty, 0]$, where $L_{\sigma m}$ denotes the Laplacian of a subgraph associated with the delay $\tau_{m}$. Clearly, $L_{\sigma}=\sum_{m=1}^{M} L_{\sigma m}$.

In this paper, the graph $\mathscr{G}$ is assumed to be connected and undirected. Then according to Lemma 1, we suppose that the eigenvalues of $L_{\sigma}$ are $0=\lambda_{1}<\lambda_{2} \leq \lambda_{3} \leq \cdots \leq \lambda_{n}$.

\section{Main Results}

In this section, we derive consensus conditions for systems $Y_{q}, q=1,2$, with a frequency domain approach. The main idea of this method is to firstly prove the consensus achievement of each system when all the time delays are equal to zero and then to calculate the delay margin to ensure that if all the delays are under the bound of it, the stability of the system will not be affected.
3.1. Consensus Conditions for the Original Systems. Firstly, we investigate the stability of $Y_{1}$ in absence of time delays. Let

$$
\Phi \triangleq I_{n} \otimes A_{1}-L_{\sigma} \otimes B_{1},
$$

and then $Y_{1}$ given by (6) $(q=1)$ with zero time delays can be written as

$$
\dot{\psi}_{1}(t)=\Phi \psi_{1}(t) .
$$

Lemma 2. The matrix $\Phi$ has a double eigenvalue at zero with a single Jordan block, and all other eigenvalues have negative real parts.

Proof. According to Lemma 1, there exists an orthogonal matrix $W$, such that

$$
W^{T} L_{\sigma} W=\operatorname{diag}\left\{0, \lambda_{2}, \ldots, \lambda_{n}\right\},
$$

and then it follows that

$$
\begin{aligned}
& \left(W \otimes I_{l}\right)^{T} \Phi\left(W \otimes I_{l}\right) \\
& \quad=\operatorname{diag}\left\{A_{1}, A_{1}-\lambda_{2} B_{1}, \ldots, A_{1}-\lambda_{n} B_{1}\right\} .
\end{aligned}
$$

Through simple calculations, we have

$$
\begin{gathered}
\operatorname{det}\left(\operatorname{diag}\left\{A_{1}, A_{1}-\lambda_{2} B_{1}, \ldots, A_{1}-\lambda_{n} B_{1}\right\}\right) \\
=s^{2} \prod_{i=2}^{n} s^{2}+\lambda_{i} k_{2} s+\lambda_{i} k_{1}=0 .
\end{gathered}
$$

The roots of each $s^{2}+\lambda_{i} k_{2} s+\lambda_{i} k_{1}=0(i \geq 2)$ are

$$
\frac{-\lambda_{i} k_{2} \pm \sqrt{\left(\lambda_{i} k_{2}\right)^{2}-4 \lambda_{i} k_{1}}}{2} \text {. }
$$

Since $\lambda_{i}>0$ when $i \geq 2$, if $\left(\lambda_{i} k_{2}\right)^{2}<4 \lambda_{i} k_{1}$, the real parts of (12) are $-\lambda_{i} k_{2} / 2<0$, and when $\left(\lambda_{i} k_{2}\right)^{2} \geq 4 \lambda_{i} k_{1}$, (12) apparently are negative real numbers. Thus the polynomial (11) has a double root at zero, and the real part of all other roots is negative; then the lemma is proven.

Remark 3. According to Lemma 2, system (8) is unstable in the sense of traditional control due to the existence of the zero Jordan block in the system matrix. However, one can easily obtain that the vectors $\mathbf{1}_{n} \otimes[1,0]^{T}$ and $\mathbf{1}_{n} \otimes[0,1]^{T}$ are the eigenvectors associated with the zero eigenvalue. Therefore the nondelayed system (8) finally converges to the space spanned by the two vectors; that is, $\lim _{t \rightarrow+\infty}\left[\zeta_{i}(t)-\zeta_{k}(t)\right]=0$, for all $i, k \in \mathscr{I}$. This has illustrated that the consensus is reached.

Reference [17] has proven that system $Y_{2}$ will achieve rotational consensus motion when all the time delays are absent. Then we summarize the results in the following lemma.

Lemma 4. Consider a network of second-order agents with fixed topology that is connected. Both of the multiagent systems $Y_{q}(q=1,2)$ given by $(6)$ will reach consensus if all the delays $\tau_{m}=0, m=1,2, \ldots, M$. 
The next step is to find out the conditions ensuring stable consensus motions of both systems.

Theorem 5. Consider the second-order systems $Y_{q}, q=1,2$, that, given by (6), the consensus problems are solved, if in each system all the delays $\tau_{m}$ are smaller than a corresponding value $\bar{\tau}_{q}, q=1,2$, where

$$
\begin{aligned}
& \text { for } Y_{1}: \bar{\tau}_{1}=\frac{\left[\arctan \left(\left(k_{2} / k_{1}\right) \bar{\omega}_{1}\right)\right]}{\bar{\omega}_{1}}, \\
& \bar{\omega}_{1}=\sqrt{\frac{\lambda_{n}^{2} k_{2}^{2}+\sqrt{\left(\lambda_{n}^{2} k_{2}^{2}\right)^{2}+4 \lambda_{n}^{2} k_{1}^{2}}}{2}} ; \\
& \text { for } Y_{2}: \bar{\tau}_{2}=\frac{\left[\pi-\arctan \left(\bar{\omega}_{2} /\left(\bar{\omega}_{2}-1\right)\right)\right]}{\bar{\omega}_{2}}, \\
& \bar{\omega}_{2}=\frac{1+\sqrt{1+4 \lambda_{n}^{2}+4 \sqrt{\lambda_{n}^{4}+\lambda_{n}^{2}}}}{2} .
\end{aligned}
$$

Proof. Consider the multiagent systems (6) with nonuniform time delays. Take $\Psi_{q}(s)$ as the Laplace transform of $\psi_{q}(t)$; we have $\Psi_{q}(s)=G_{\tau q}^{-1} \Psi_{q}(0)$, where

$$
G_{\tau q}(s)=s I_{2 n}-\left(I_{n} \otimes A_{q}\right)+\sum_{m=1}^{M}\left(L_{\sigma m} \otimes B_{q}\right) e^{-\tau_{m} s}
$$

for $q=1,2$. According to the foregoing discussions, to study the stability of the delayed system, we only need to investigate the values of $\tau_{m}$ that guarantee the existence of nonzero roots of $G_{\tau q}(s)$ on imaginary axis, which represents the crossing of the characteristic roots from the stable region to the unstable one. The roots of characteristic polynomials such as $G_{\tau q}(s)$ are hereinafter referred to as "the eigenvalues of the system."

Suppose that, for each $q \in\{1,2\}, s_{q}=j \omega_{q} \neq 0$ is an imaginary root of $G_{\tau q}(s)$, and $u_{q}=u_{q 1} \otimes[1,0]^{T}+u_{q 2} \otimes[0,1]^{T}$ is a corresponding eigenvector, where $\left\|u_{q}\right\|=1, u_{q 1}, u_{q 2} \in \mathbb{C}^{n}$. Then we have

$$
\begin{aligned}
& {\left[j \omega_{q} I_{2 n}-\left(I_{n} \otimes A_{q}\right)+\sum_{m=1}^{M}\left(L_{\sigma m} \otimes B_{q}\right) e^{-j \omega_{q} \tau_{m}}\right] u_{q}} \\
& \quad=0 .
\end{aligned}
$$

Note that all the complex roots of each $G_{\tau q}(s)$ appeared in conjugated pairs; we only need to study the situation that $\omega_{q}>$ 0 . Since the elements of the vector obtained by calculating the left part of (15) equal zero, we have

$$
\begin{aligned}
j \omega_{1} u_{11} & =u_{12}, \\
\left(1-\omega_{2}\right) u_{21} & =u_{22} .
\end{aligned}
$$

Multiplying by $u_{q}^{H}$ (the conjugate transpose of $u_{q}$ ) on the left side of the left part of (15), and with (16) substituted, it yields

$$
\begin{aligned}
& \sum_{m=1}^{M} \alpha_{m} e^{-j \omega_{1} \tau_{m}}=\frac{\omega_{1}^{2}}{k_{1}+j \omega_{1} k_{2}}, \\
& \sum_{m=1}^{M} \alpha_{m} e^{-j \omega_{2} \tau_{m}}=\frac{-j \omega_{2}\left(\omega_{2}-1\right)}{\omega_{2}+j\left(\omega_{2}-1\right)},
\end{aligned}
$$

where

$$
\alpha_{m}=\frac{u_{q}^{H}\left(L_{\sigma m} \otimes I_{2}\right) u_{q}}{u_{q}^{H} u_{q}} .
$$

Equation (17) can be rewritten as

$$
\begin{aligned}
& \sum_{m=1}^{M} \alpha_{m} e^{j \omega_{1} \tau_{m}}=\frac{\omega_{1}^{2}}{k_{1}-j \omega_{1} k_{2}} \triangleq F_{1}\left(\omega_{1}\right), \\
& \sum_{m=1}^{M} \alpha_{m} e^{j \omega_{2} \tau_{m}}=\frac{j \omega_{2}\left(\omega_{2}-1\right)}{\omega_{2}-j\left(\omega_{2}-1\right)} \triangleq F_{2}\left(\omega_{2}\right) .
\end{aligned}
$$

Take modulus of both sides of (19) to obtain

$$
\begin{aligned}
M_{q}\left(\omega_{q}\right) & \triangleq\left|F_{q}\left(\omega_{q}\right)\right|=\left|\sum_{m=1}^{M} \alpha_{m} e^{j \omega_{q} \tau_{m}}\right| \leq\left|\sum_{m=1}^{M} \alpha_{m}\right| \\
& =\frac{u_{q}^{H}\left(L_{\sigma} \otimes I_{l}\right) u_{q}}{u_{q}^{H} u_{q}} \leq \lambda_{n},
\end{aligned}
$$

for $q=1,2$. After some calculation, we find that the inequality $M_{q}\left(\omega_{q}\right) \leq \lambda_{n}$ from (20) holds true if and only if $\omega_{q} \leq \bar{\omega}_{q}$. This is a necessary condition for our hypothesis to hold.

The idea now is to analyze the argument of each $F_{q}\left(\omega_{q}\right)$, to find out how $\tau_{m}$ affects the validity of our hypothesis that the imaginary eigenvalues $j \omega_{q}$ exist. Calculate the arguments of $F_{1}\left(\omega_{q}\right)$; we get

$$
\begin{aligned}
& \theta_{1}\left(\omega_{1}\right) \triangleq \arg \left[F_{1}\left(\omega_{1}\right)\right]=\arctan \left(\frac{k_{2}}{k_{1}} \omega_{2}\right), \\
& \theta_{2}\left(\omega_{2}\right) \triangleq \arg \left[F_{2}\left(\omega_{2}\right)\right] \\
&= \begin{cases}\arctan \left(\frac{\omega_{2}}{1-\omega_{2}}\right), & \omega_{2}<1, \\
\frac{\pi}{2} & \omega_{2}=1, \\
\pi-\arctan \left(\frac{\omega_{2}}{\omega_{2}-1}\right), & \omega_{2}>1,\end{cases}
\end{aligned}
$$

where $\theta_{q}\left(\omega_{1}\right) \in[0,2 \pi)$. Define $\tau_{q}\left(\omega_{q}\right) \triangleq \theta_{q}\left(\omega_{q}\right) / \omega_{q}$. Then the following proves that, for each $q=1,2, \bar{\tau}_{q}=\tau_{q}\left(\bar{\omega}_{q}\right)$ is 
the minimal value of $\tau_{q}\left(\omega_{q}\right)$ when $\omega_{q} \leq \bar{\omega}_{q}$. For system $Y_{1}$, Let $a \triangleq k_{2} / k_{1}$. Calculate derivative of $\tau_{1}\left(\omega_{1}\right)$ :

$$
\begin{aligned}
D_{1}\left(\omega_{1}\right) & \triangleq \frac{d\left(\tau_{1}\left(\omega_{1}\right)\right)}{d \omega_{1}} \\
& =\frac{a}{\omega_{1}\left(a^{2} \omega_{1}^{2}+1\right)}-\frac{\arctan \left(a \omega_{1}\right)}{\omega_{1}^{2}} \\
& =\frac{1}{\omega_{1}^{2}} \underbrace{\left[\frac{a \omega_{1}}{a^{2} \omega_{1}^{2}+1}-\arctan \left(a \omega_{1}\right)\right]}_{D_{2}\left(\omega_{1}\right)},
\end{aligned}
$$

and the derivative of $D_{2}\left(\omega_{1}\right)$ is

$$
\frac{d\left(D_{2}\left(\omega_{1}\right)\right)}{d \omega_{1}}=-\frac{2 a^{3} \omega_{1}^{2}}{\left(a^{2} \omega_{1}^{2}+1\right)^{2}}<0 .
$$

Thus $D_{2}\left(\omega_{1}\right)<D_{2}(0)=0$ when $\omega_{1}>0$. Consequently, $D_{1}\left(\omega_{1}\right)=D_{2}\left(\omega_{1}\right) / \omega_{1}^{2}<0$ when $\omega_{1}>0$, which means $\tau_{1}\left(\omega_{1}\right)$ decreases as $\omega_{1}$ grows. Therefore we have $\bar{\tau}_{1}=\tau_{1}\left(\bar{\omega}_{1}\right) \leq$ $\tau_{1}\left(\omega_{1}\right)$, when $\omega_{1} \leq \bar{\omega}_{1}$. For system $Y_{2}$, when $\omega_{2}<1$, one can easily calculate that $\tau_{2}\left(\omega_{2}\right)>1$ always holds; and when $\omega_{2} \geq$ $1, \tau_{2}\left(\omega_{2}\right)$ is monotonically decreasing from $\pi / 2$; moreover, when $\omega>2, \tau_{2}\left(\omega_{2}\right)<1$ (we omit the proof which is similar to the one given above). Because the network of the system is connected, $\lambda_{n}>1$ holds true; it follows that $\bar{\omega}_{2}>2$. Therefore, one cannot find a smaller value of $\tau_{2}\left(\omega_{2}\right)$ than $\bar{\tau}_{2}=\tau_{2}\left(\bar{\omega}_{2}\right)$ when $\omega_{2} \leq \bar{\omega}_{2}$.

Note that $\theta_{q}\left(\omega_{q}\right)$ are also the arguments of the left parts of (19); if for each system we set all $\tau_{m}<\bar{\tau}_{q}$, we have

$$
\begin{aligned}
\tau_{q}\left(\omega_{q}\right) & =\frac{\theta_{q}\left(\omega_{q}\right)}{\omega_{q}}=\frac{\arg \left(\sum_{m=1}^{M} \alpha_{m} e^{j \omega_{q} \tau_{m}}\right)}{\omega_{q}} \\
& \leq \frac{\max \left\{\omega_{q} \tau_{m}\right\}}{\omega_{q}}<\frac{\omega_{q} \bar{\tau}_{q}}{\omega_{q}}=\bar{\tau}_{q},
\end{aligned}
$$

for $q=1,2$, which conflicts with the idea that $\bar{\tau}_{q}=\tau_{q}\left(\bar{\omega}_{q}\right)$ is the minimal value of $\tau_{q}\left(\omega_{q}\right)$ when $\omega_{q} \leq \bar{\omega}_{q}$; and this rejects our hypothesis. Consequently, each of the two systems has no nonzero imaginary eigenvalue when all the delays $\tau_{m}<$ $\bar{\tau}_{q}$. Following the continuity of function, the zero crossing of eigenvalues from the left half plane to the right half plane has been prevented, and the bounded delays will not bring about instabilities to the systems. According to Lemma 4, the system can reach consensus with bounded delays, and the theorem is proven.

In fact, for each system, if all $\tau_{m}=\bar{\tau}_{q}$, then there must exist an imaginary eigenvalue $j \bar{\omega}_{q}$ and corresponding eigenvector $u_{q}$ that satisfy $\left|\sum_{m=1}^{M} \alpha_{m}\right|=\lambda_{n}$. That is, according to (19),

$$
\sum_{m=1}^{M} \alpha_{m} e^{j \bar{\omega}_{q} \bar{\tau}_{q}}=F_{q}\left(\bar{\omega}_{q}\right)
$$

holds true.
Remark 6. $\bar{\tau}_{q}$ are termed the delay margins. If we draw trajectories of the delays $\tau_{m}$ that each value grows from 0 to $+\infty$, then the touch of $\tau_{m}=\bar{\tau}_{q}$ leads to the first contact of the eigenvalues from the stable region to the unstable one. When all the delays exceed $\bar{\tau}_{q}$, the system will be unstable, because there must exist at least one eigenvalue in the unstable region (the RHP). The instabilities get worse with increasing $\tau_{m}$.

Remark 7. Reference [9] has provided a general method of obtaining delay margins for delayed systems (including nonuniform delays) to solve consensus problems, and the method proposed in this paper is inspired by that idea. However, only first-order systems have been considered in [9], where the arguments of $\sum \alpha_{m} e^{j \omega_{q} \tau_{m}}$ are bounded by the simple $\pi / 2$. In our paper, the arguments $\theta_{q}\left(\omega_{q}\right)$ are bounded by variables changing with $\omega_{q}$; thus we have to find possible maximal delay values related to $\omega_{q}$ to cause the contradictions. Reference [21] has discussed the consensus problem of multiagent systems with delays using frequency approaches, but the agent dynamics are only considered in the form of single integrator, and the methods can not be directly used in second-order systems. In the literatures [11, 22], the authors studied the consensus problems of second-order systems with uniform delays; however, the situations of multiple time delays were not discussed. In [18], the stability analyses of second-order multiagent systems with nonuniform delays were performed using Lyapunov methods, and the results were presented in the form of Linear Matrix Inequalities (LMIs).

3.2. Consensus Phenomenon of System $Y_{1}$ without Input Delays. In many cases, the consensus is reached when all agents converge on a point and stay. References [11, 19] have proposed consensus algorithms enabling the vehicles to drive together at a uniform velocity after their convergence. These classical algorithms are then extensively applied in the subsequent works, such as the generalized use in [23] to solve the tracking problem. Therefore exploring such basic algorithms is very useful and the results can be generalized for many applications. In this paper, the idea of $Y_{1}$ comes from the algorithm proposed in [11]; but we consider nonuniform time delays. In our systems, each agent gets delayed information from its neighbors, and besides these communication delays, input delays also occur. Consequently, one may come up with a question that what if the input delays are absent while communication delays still occur. In this situation, the control input of (1) for $Y_{1}$ becomes

$$
\begin{aligned}
& u_{i}(t)=\sum_{s_{k} \in N_{i}} a_{i k}\left\{k_{1}\left[x_{i}(t)-x_{k}\left(t-\tau_{i k}\right)\right]\right. \\
& \left.+k_{2}\left[v_{i}(t)-v_{j}\left(t-\tau_{i k}\right)\right]\right\} .
\end{aligned}
$$

Suppose that there is a time when all the agents are moving forward together at the same point; then the value of $x_{i}(t)-x_{k}\left(t-\tau_{i k}\right)>0$ depends on the value of $\tau_{i k}$ and how fast the agents move. If this happens, (26), which determines the acceleration of each agent, will not equal zero, and it is difficult for $u_{i}(t)$ and $u_{k}(t)(i \neq k)$ to reach the same value due 
to the nonuniform delays. Then, in the next period of time, the agents have to separate from each other because of their different accelerations.

Simulations (see in Section 4) have shown a reasonable consensus phenomenon when applying (26) to the system: when appropriate delays are set to the system, the agents move to get together at first, and then slow down until they stop at the same point, which means that the agents will not finally achieve a collective motion when the input delays are absent while communication delays still occur.

3.3. Conditions for an Expanded System to Achieve Rotational Consensus Motion. System $Y_{2}$ given by $(6)(q=2)$ has provided a solution that the agents are intended to perform a rotational consensus motion in the complex plane. However, in many practical cases, the processors of the systems do not accept complex numbers directly; as an example, it is difficult to draw a simulation model in the MATLAB for such a system involving complex state variables. Commonly, we "expand" the system by replacing each complex state variable with two real variables: the real part and the imaginary part of the original variable, respectively. The new system is equipped with real coefficient matrices.

Denote the new state vector and coefficient matrices:

$$
\begin{aligned}
& \overline{\psi_{2}}(t) \triangleq\left[\operatorname{Re}\left(x_{1}\right), \operatorname{Im}\left(x_{1}\right), \operatorname{Re}\left(c_{1}\right), \operatorname{Im}\left(c_{1}\right), \operatorname{Re}\left(x_{2}\right),\right. \\
& \quad \operatorname{Im}\left(x_{2}\right), \operatorname{Re}\left(c_{2}\right), \operatorname{Im}\left(c_{2}\right), \ldots, \operatorname{Re}\left(x_{n}\right), \operatorname{Im}\left(x_{n}\right), \operatorname{Re}\left(c_{n}\right), \\
& \left.\quad \operatorname{Im}\left(c_{n}\right)\right] \\
& \overline{A_{2}} \triangleq \operatorname{Re}\left(A_{2}\right) \otimes I_{2}+\operatorname{Im}\left(A_{2}\right) \otimes E_{2}, \\
& \overline{B_{2}} \triangleq \operatorname{Re}\left(B_{2}\right) \otimes I_{2}+\operatorname{Im}\left(B_{2}\right) \otimes E_{2},
\end{aligned}
$$

where $\operatorname{Re}(\cdot)$ and $\operatorname{Im}(\cdot)$ mean the real and imaginary part of the term enclosed, respectively, and

$$
E_{2} \triangleq\left[\begin{array}{cc}
0 & -1 \\
1 & 0
\end{array}\right]
$$

We get the new system expanded from the system given by $(6)(q=2)$ :

$$
\begin{aligned}
\dot{\overline{\psi_{2}}}(t)= & \left(I_{n} \otimes \overline{A_{2}}\right) \overline{\psi_{2}}(t) \\
& -\sum_{m=1}^{M}\left(L_{\sigma m} \otimes \overline{B_{2}}\right) \overline{\psi_{2}}\left(t-\tau_{m}\right) .
\end{aligned}
$$

Equation (29) is another form of $Y_{2}$, and its state variables are real values. Agents in system (29) behave like those in $Y_{2}$ given by (6) $(q=2)$ when all the time delays are absent. Therefore, in the light of the real state variables, we usually simulate the expanded system instead of the original one. However, there is a notable difference between the two systems. The amount of eigenvalues of the new system (29) doubles that of (6) ( $q=$ $2)$. We introduce another related system for reference:

$$
\begin{aligned}
\dot{\psi}_{2}(t)= & \left(I_{n} \otimes A_{2}^{*}\right) \psi_{2}(t) \\
& -\sum_{m=1}^{M}\left(L_{\sigma m} \otimes B_{2}^{*}\right) \psi_{2}\left(t-\tau_{m}\right),
\end{aligned}
$$

where $A_{2}^{*}$ and $B_{2}^{*}$ are the the conjugate matrices of $A_{2}$ and $B_{2}$, respectively. The subsequent lemma illustrates the relationships among the three systems $(6)(q=2),(30)$, and (29).

Lemma 8. There are $4 n$ eigenvalues of system (29), which consist of all the $2 n$ eigenvalues of $(6)(q=2)$ and all the $2 n$ eigenvalues of (30).

Proof. Suppose that $\gamma$ is one eigenvalue of $(6)(q=2)$, and the corresponding eigenvector is $\alpha$. Then we have

$$
\left[\gamma I_{2 n}-\left(I_{n} \otimes A_{2}\right)+\sum_{m=1}^{M}\left(L_{\sigma m} \otimes B_{2}\right) e^{-\tau_{m} \gamma}\right] \alpha=0 .
$$

Substituting $\gamma$ into the characteristic polynomial of system (29), and multiplying by $\alpha \otimes[j, 1]$, it yields

$$
\begin{aligned}
& {\left[\gamma I_{4 n}-\left(I_{n} \otimes \overline{A_{2}}\right)+\sum_{m=1}^{M}\left(L_{\sigma m} \otimes \overline{B_{2}}\right) e^{-\tau_{m} \gamma}\right]\{\alpha} \\
& \otimes[j, 1]\} \\
& =\left\{\left[\gamma I_{2 n}-\left(I_{n} \otimes A_{2}\right)+\sum_{m=1}^{M}\left(L_{\sigma m} \otimes B_{2}\right) e^{-\tau_{m} \gamma}\right] \alpha\right\} \\
& \otimes[j, 1]=0,
\end{aligned}
$$

which implies that $\gamma$ is an eigenvalue of system (29), and $\alpha \otimes[j, 1]$ a corresponding eigenvector. It follows that the eigenvalues of $(6)(q=2)$ also are the eigenvalues of (29). By the same way, one can prove that each eigenvalue of system (30), whose corresponding eigenvector is here denoted as $\alpha_{p}$, is also an eigenvalue of (29), and $\alpha_{p} \otimes[-j, 1]$ can be a corresponding eigenvector. As the $2 n+2 n=4 n$ eigenvalues fit the system (29) in amount, and (29) has no other eigenvalues, the lemma is proven.

Lemma 9. The expanded multiagent system (29) with real state variables will reach consensus if all the time delays are absent; that is, $\tau_{m}=0, m=1,2, \ldots, M$.

Proof. When all the time delays equal zero, the eigenvalues of system (30) are the conjugate numbers of the eigenvalues of (6) $(q=2)$. As each conjugated pair has the same real parts, the expanded system (29) has the same stability properties as (6) $(q=2)$ when all time delays are absent, and the agents will achieve rotational consensus motion. 
Theorem 10. Considering the multiagent system (29) expanded from $(6)(q=2)$, it will reach consensus if all the delays $\tau_{m}<\bar{\tau}$, where

$$
\begin{aligned}
\bar{\tau} & =\min \left\{\tau_{d 1}, \tau_{d 2}\right\} \\
\tau_{d 1} & =\frac{\left[\pi-\arctan \left(\omega_{d 1} /\left(\omega_{d 1}-1\right)\right)\right]}{\omega_{d 1}}, \\
\omega_{d 1} & =\frac{1+\sqrt{1+4 \lambda_{n}^{2}+4 \sqrt{\lambda_{n}^{4}+\lambda_{n}^{2}}}}{2}, \\
\tau_{d 2}= & \frac{\left[\arctan \left(\omega_{d 2} /\left(\omega_{d 2}+1\right)\right)\right]}{\omega_{d 2}}, \\
\omega_{d 2}= & \frac{-1+\sqrt{1+4 \lambda_{n}^{2}+4 \sqrt{\lambda_{n}^{4}+\lambda_{n}^{2}}}}{2} .
\end{aligned}
$$

Proof. Theorem 5 has proven that $\tau_{d 1}$ is the delay margin ensuring that the eigenvalues of $(6)(q=2)$ are in the stable region. We can also prove that the eigenvalues of system (30) are in the stable region if all the delays are bounded by $\tau_{d 2}$, and the system will be unstable if all the delays exceed $\tau_{d 2}$, by the same way as in the proof of Theorem 5. Thus the proof is omitted.

The foregoing discussions have helped us to understand that when all the delays $\tau_{m}<\bar{\tau}$, the eigenvalues of both systems $(6)(q=2)$ and $(30)$ are in the stable region. According to Lemmas 8 and 9, system (30) can reach consensus with nonuniform time delays bounded by $\bar{\tau}$.

Remark 11. The theorem above has provided us with a delay bound ensuring the consensus achievement. Note that both $\tau_{d 1}$ and $\tau_{d 2}$ are delay margins of the corresponding systems. We set all the time delays of the expanded system (29) greater than $\bar{\tau}$; when these delays are put into the systems (6) $(q=$ 2 ) and (30), at least one of the systems will have eigenvalues in the unstable region; this will bring about the failure of the expanded system (29) to achieve consensus motion. Hence $\bar{\tau}$ is also the delay margin for the expanded system.

\section{Numerical Examples}

In this section, some simulations are carried out to evidence the theoretical results obtained by the previous analysis.

Consider that a multiagent system consists of 4 agents, whose communication topology is described in graph $\mathscr{G}$ shown in Figure 1. The 1st-2nd and 3rd-4th agent pairs are connected and the communication delay between each of them is $\tau_{1}$; the 2 nd-3rd and 4 th-1st agent pairs are connected in presence of communication delay $\tau_{2}$. Suppose the weight of each edge on $\mathscr{G}$ is 1 ; then $\lambda_{n}=4$.

We start with $Y_{1}$ given by $(6)(q=1)$. Let $k_{1}=1.2$ and $k_{2}=1$; then according to Theorem 5, the delay bound is $\bar{\tau}=0.31$. Firstly, we set the nonuniform time delays labelled on graph $\mathscr{G}$ as $\tau_{1}(t)=0.30$, and $\tau_{2}(t)=0.29$, which are under the bound. Apparently, from the simulation results shown in

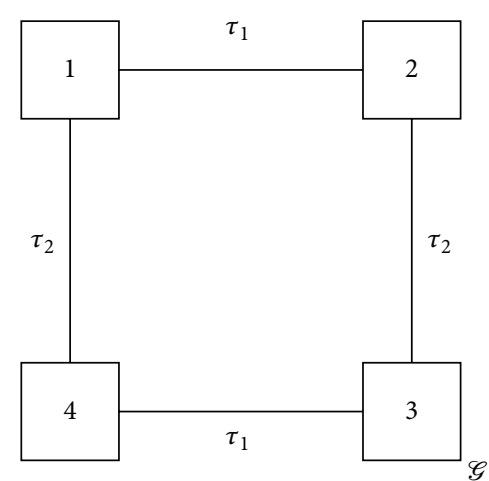

Figure 1: The communication topology of the 4-agent system.

Figure 2, the consensus is reached. As a contrast example, we change the value of delays to see whether the agents could reach consensus again when the delays exceed the bound. Here $\tau_{1}(t)=0.31$ and $\tau_{2}(t)=0.32$. Figure 3 has shown that the agents fail to reach consensus.

To understand what actually happens to the multiagent system when (26) is the control input of system (1), we set $k_{1}=1.2, k_{2}=1$, and the time delays $\tau_{1}(t)=0.01, \tau_{2}(t)=$ 0.04. The simulation results shown in Figure 4 indicate the situation that is described in Section 3.2.

For the system to achieve rotational consensus motion, we use the expanded system (29) as the simulation model. According to Theorem 10, $\bar{\tau}=0.134$. We also execute the simulation twice: for the first time, we set $\tau_{1}(t)=$ $0.12, \tau_{2}(t)=0.13$ as the bounded delays; and for the second time, the delays are set as $\tau_{1}(t)=0.14, \tau_{2}(t)=0.15$ which exceed the delay margin. The results are shown in the first and second pictures in Figure 5 separately, and they have validated Theorem 10.

\section{Conclusions}

In this work, we studied two types of consensus problems of multiagent systems with nonuniform time delays: one makes the agents achieve a collective rectilinear motion, and the other demands the agents to finally move rotationally. We provided sufficient conditions in the form of delay margins for the systems to reach consensus and carried out simulations to show the instance of the results.

Frequency domain approaches are commonly limited to linear systems. The proposed method is not compatible with nonlinear systems. Further research will focus on consensus problems of delayed multiagent systems with high-order dynamics and directed topologies. High-order dynamics will cause the occurrence of high-order polynomials in (20); directed topologies will bring complex eigenvalues to Laplacian $L_{\sigma}$ and break monotonicity of $\theta_{q}\left(\omega_{q}\right)$. Nevertheless, it is realistic to overcome these difficulties and adapt the approach to such systems in the future as we explore proper mathematical tools. 

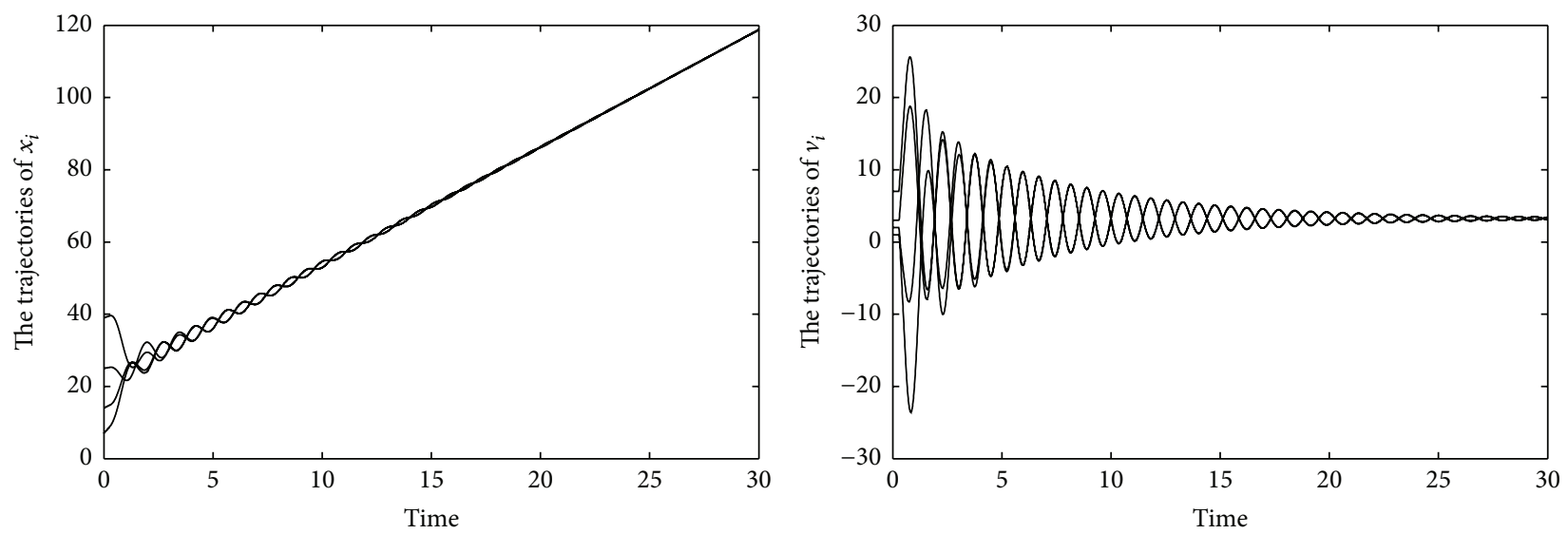

Figure 2: The trajectories of the position and velocity in $Y_{1}$ given by $(6)(q=1)$ when delays are under the bound.
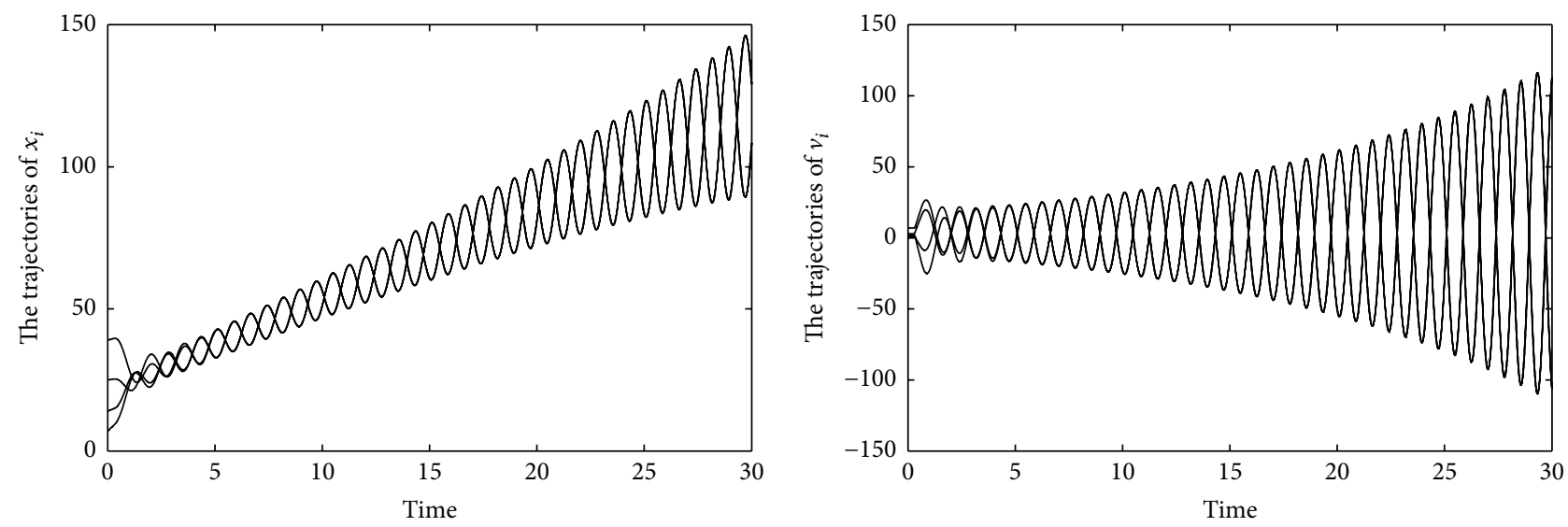

FIGURE 3: The trajectories of the position and velocity in $Y_{1}$ given by $(6)(q=1)$ when delays exceed the bound.
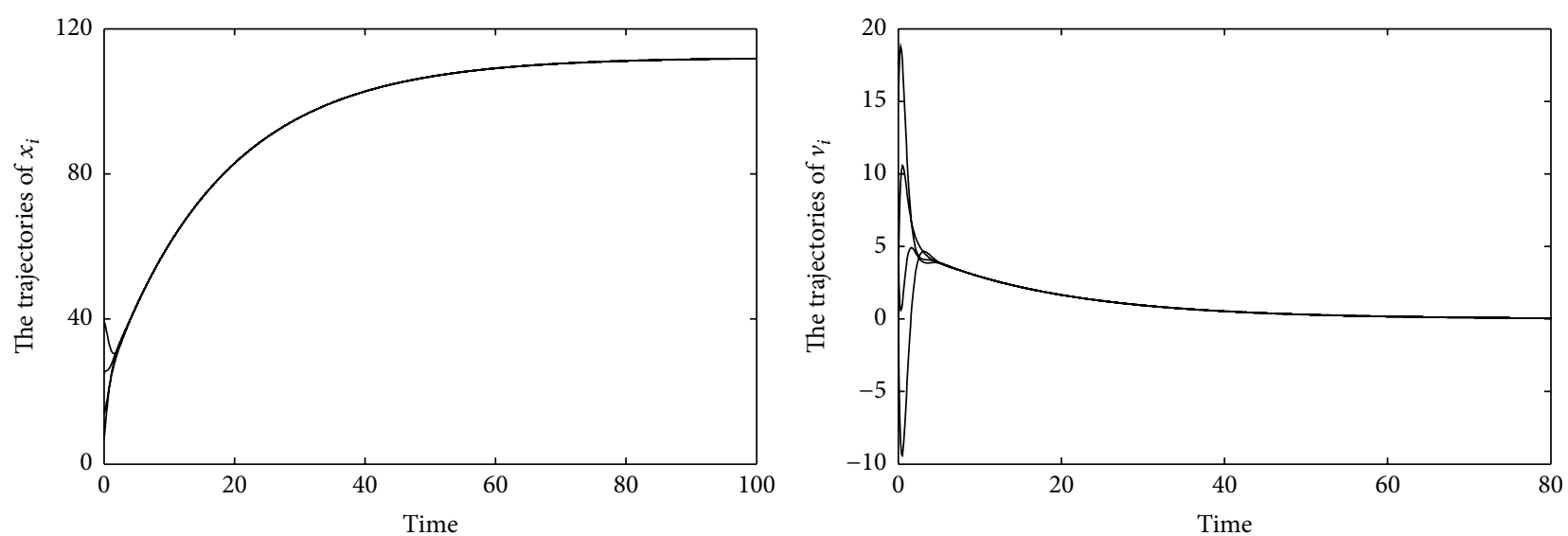

FIGURE 4: The trajectories of the position and velocity in the system (1) applying control input (26) where input delays are absent. 

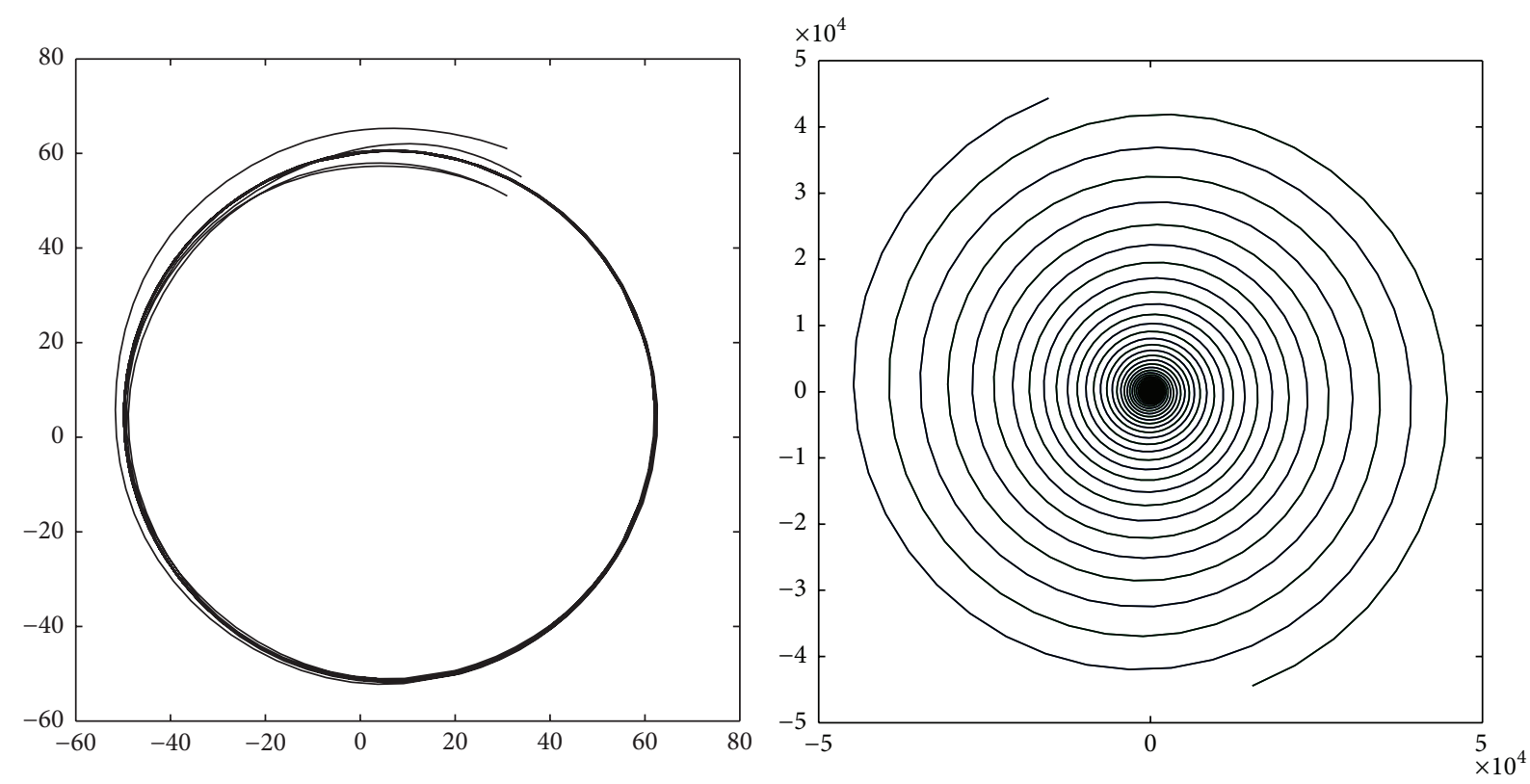

FIGURE 5: The trajectories of the agents in the system to achieve rotational consensus motion given by (29).

\section{Competing Interests}

The authors declare that they have no competing interests.

\section{Acknowledgments}

This work has been supported by the Specialized Research Fund for the Doctoral Program of Higher Education (20130185110023).

\section{References}

[1] J. A. Fax and R. M. Murray, "Information flow and cooperative control of vehicle formations," IEEE Transactions on Automatic Control, vol. 49, no. 9, pp. 1465-1476, 2004.

[2] Y. Hong, J. Hu, and L. Gao, "Tracking control for multiagent consensus with an active leader and variable topology," Automatica, vol. 42, no. 7, pp. 1177-1182, 2006.

[3] R. Olfati-Saber and J. S. Shamma, "Consensus filters for sensor networks and distributed sensor fusion," in Proceedings of the 44th IEEE Conference on Decision and Control, and the European Control Conference (CDC-ECC '05), pp. 6698-6703, IEEE, Seville, Spain, December 2005.

[4] H. Meng, Z. Chen, L. Zhu, and R. Middleton, "Consensus of a class of second-order nonlinear heterogeneousmulti-agent systems with uncertainty and communication delay," International Journal of Robust and Nonlinear Control, 2016.

[5] B. Mirkin, P.-O. Gutman, and Y. Shtessel, "Asymptotic sliding mode control approach to adaptive distributed tracking problem for multi-agent nonlinear delayed systems," International Journal of Control, vol. 85, no. 11, pp. 1671-1682, 2012.

[6] U. Münz, A. Papachristodoulou, and F. Allgöwer, "Generalized Nyquist consensus condition for high-order linear multi-agent systems with communication delays," in Proceedings of the 48th IEEE Conference on Decision and Control held jointly with the 28th Chinese Control Conference (CDC/CCC '09), pp. 47654771, Shanghai, China, December 2009.

[7] P. Li, K. Qin, and M. Shi, "Distributed robust $H_{\infty}$ rotating consensus control for directed networks of second-order agents with mixed uncertainties and time-delay," Neurocomputing, vol. 148, pp. 332-339, 2015.

[8] P. Lin and Y.-M. Jia, "Multi-agent consensus with diverse timedelays and jointly-connected topologies," Automatica, vol. 47, no. 4, pp. 848-856, 2011.

[9] P.-A. Bliman and G. Ferrari-Trecate, "Average consensus problems in networks of agents with delayed communications," Automatica, vol. 44, no. 8, pp. 1985-1995, 2008.

[10] J. Wang, Q. Zhang, and H. Li, "Delay consensus of second-order nonlinear leader-following multi-agent systems," in Proceedings of the 2015 Chinese Intelligent Systems Conference, Y. Jia, J. Du, $\mathrm{H}$. Li, and W. Zhang, Eds., vol. 1 of Lecture Notes in Electrical Engineering, pp. 615-623, Springer, New York, NY, USA, 2016.

[11] P. Lin, Y. Jia, J. Du, and S. Yuan, "Distributed consensus control for second-order agents with fixed topology and time-delay," in Proceedings of the 26th Chinese Control Conference (CCC '07), pp. 577-581, July 2007.

[12] M. J. Park, O. M. Kwon, J. H. Park, S. M. Lee, and E. J. Cha, "Leader-following consensus criteria for multi-agent systems with time-varying delays and switching interconnection topologies," Chinese Physics B, vol. 21, no. 11, Article ID 110508, 2012.

[13] P. Deshpande, P. P. Menon, C. Edwards, and I. Postlethwaite, "Formation control of multi-agent systems with double integrator dynamics using delayed static output feedback," in Proceedings of the 50th IEEE Conference on Decision and Control and European Control Conference (CDC-ECC '11), pp. 34463451, IEEE, Orlando, Fla, USA, December 2011.

[14] P. Lin and W. Ren, "Constrained consensus in unbalanced networks with communication delays," IEEE Transactions on Automatic Control, vol. 59, no. 3, pp. 775-781, 2014.

[15] P. Lin and Y.-M. Jia, "Consensus of second-order discretetime multi-agent systems with nonuniform time-delays and 
dynamically changing topologies," Automatica, vol. 45, no. 9, pp. 2154-2158, 2009.

[16] Y. Pei and J. Sun, "Consensus of discrete-time linear multiagent systems with Markov switching topologies and timedelay," Neurocomputing, vol. 151, no. 2, pp. 776-781, 2015.

[17] P. Lin and Y.-M. Jia, "Distributed rotating formation control of multi-agent systems," Systems \& Control Letters, vol. 59, no. 10, pp. 587-595, 2010.

[18] A. Seuret, D. V. Dimarogonas, and K. H. Johansson, "Consensus under communication delays," in Proceedings of the 47th IEEE Conference on Decision and Control (CDC '08), pp. 4922-4927, IEEE, Cancun, Mexico, December 2008.

[19] W. Ren and E. Atkins, "Distributed multi-vehicle coordinated control via local information exchange," International Journal of Robust and Nonlinear Control, vol. 17, no. 10-11, pp. 1002-1033, 2007.

[20] C. D. Godsil, G. Royle, and C. Godsil, Algebraic Graph Theory, vol. 8, Springer, New York, NY, USA, 2001.

[21] Y.-P. Tian and C.-L. Liu, "Consensus of multi-agent systems with diverse input and communication delays," IEEE Transactions on Automatic Control, vol. 53, no. 9, pp. 2122-2128, 2008.

[22] C.-L. Liu and F. Liu, "Dynamical consensus seeking of secondorder multi-agent systems based on delayed state compensation," Systems and Control Letters, vol. 61, no. 12, pp. 1235-1241, 2012.

[23] L. Li and H. Fang, "Bounded consensus tracking for secondorder multi-agent systems with communication delay and sampled information," in Proceedings of the International Conference on Modelling, Identification and Control (ICMIC '12), pp. 584589, June 2012. 


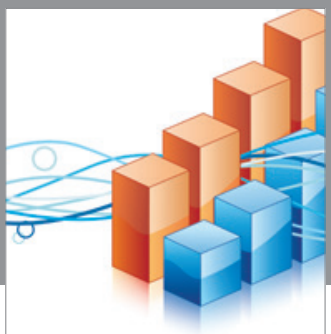

Advances in

Operations Research

vatem alat4

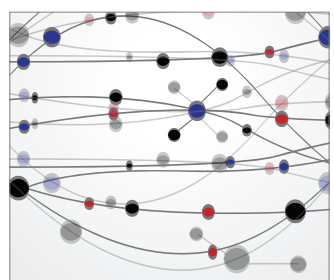

\section{The Scientific} World Journal
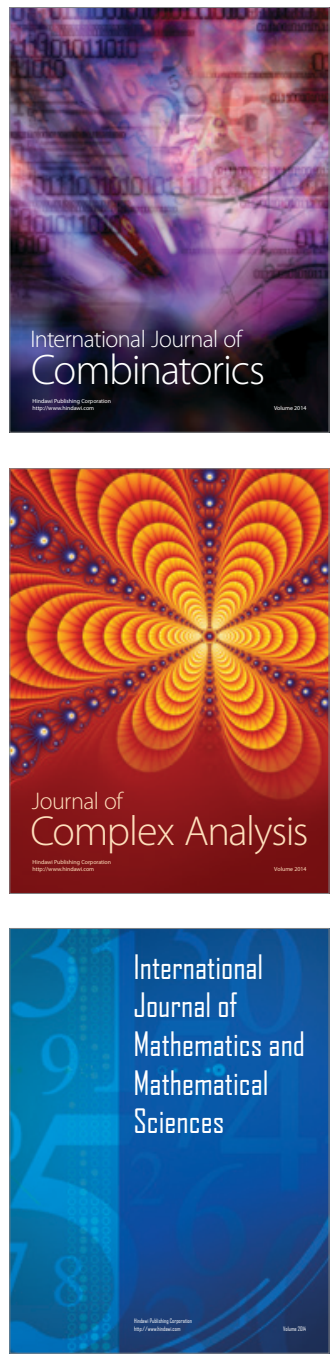
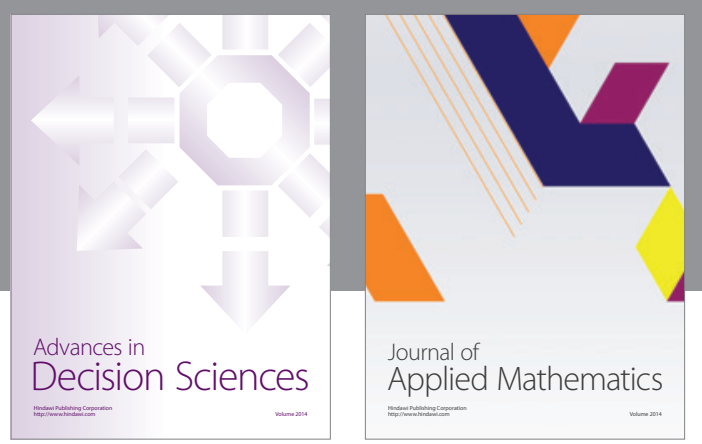

Algebra

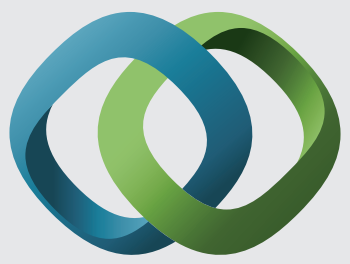

\section{Hindawi}

Submit your manuscripts at

http://www.hindawi.com
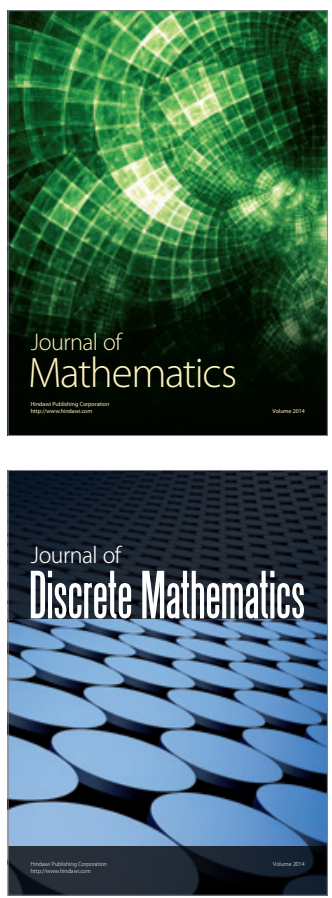

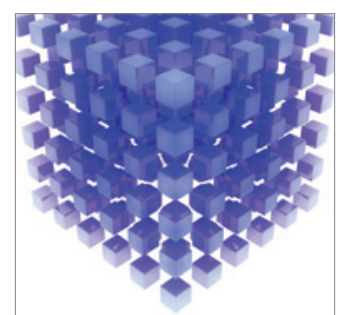

Mathematical Problems in Engineering
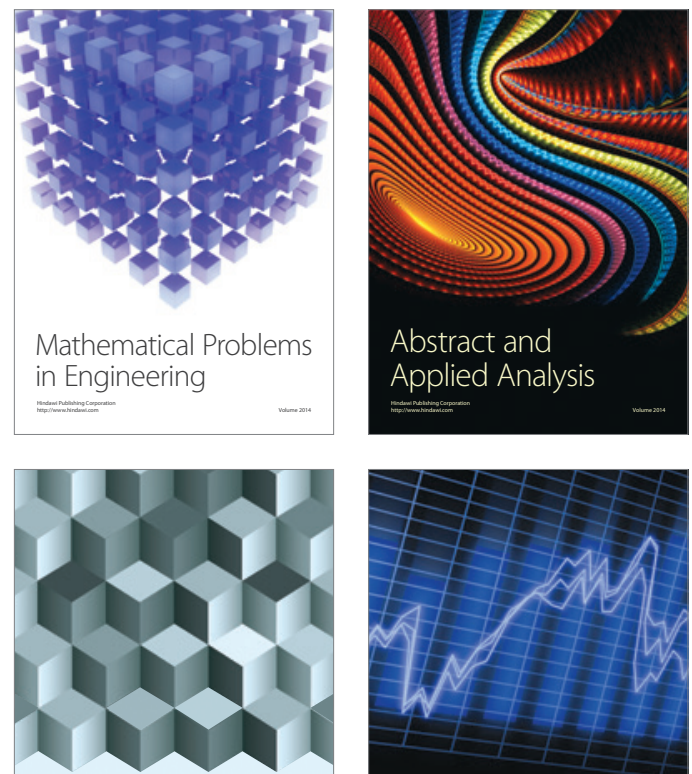

Journal of

Function Spaces

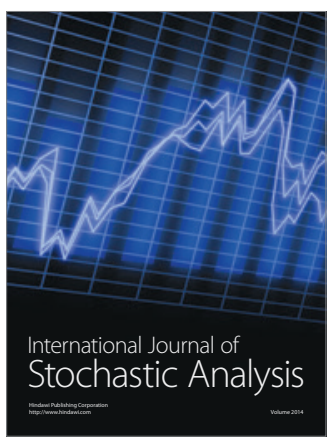

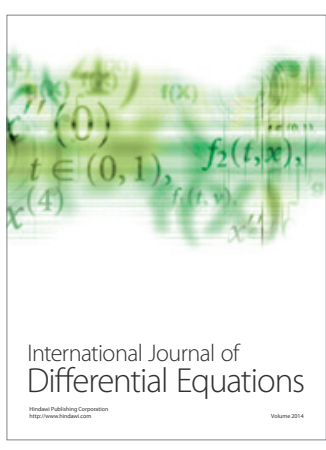
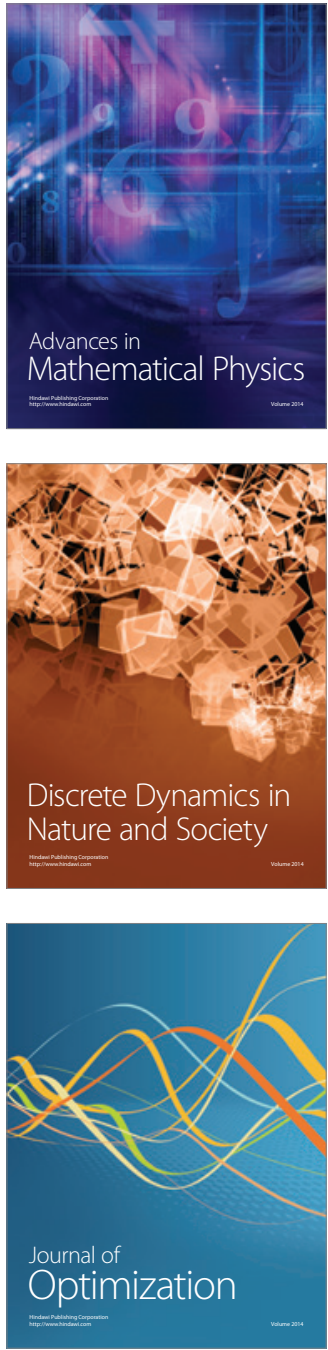